\title{
Planar subgraphs without low-degree nodes
}

\section{Kranakis, Evangelos}

Springer-Verlag

2011

Kranakis , E , Morales Ponce , O \& Suomela , J 2011, Planar subgraphs without low-degree nodes . in F Dehne , J lacono \& J-R Sack (eds), Algorithms and Data Structures : 12th International Symposium, WADS 2011. New York, NY, USA, August 15-17, 2011. Proceedings . Lecture Notes in Computer Science, vol. 6844, Springer-Verlag, pp. pÿ583 594 , Algorithms and Data Structures Symposium (WADS) , New York, NY, United States , 15/08/2011. https://doi.org/10.1007/978-3-642-22300-6_49

http://hdl.handle.net/10138/28214

https://doi.org/10.1007/978-3-642-22300-6_49

acceptedVersion

Downloaded from Helda, University of Helsinki institutional repository.

This is an electronic reprint of the original article.

This reprint may differ from the original in pagination and typographic detail.

Please cite the original version. 


\title{
Planar Subgraphs without Low-Degree Nodes
}

\author{
Evangelos Kranakis ${ }^{1}$, Oscar Morales Ponce ${ }^{1}$, and Jukka Suomela ${ }^{2}$ \\ 1 School of Computer Science, Carleton University, Ottawa, Canada \\ kranakis@scs.carleton. ca, omponce@connect.carleton.ca \\ ${ }^{2}$ Helsinki Institute for Information Technology HIIT, University of Helsinki, Finland \\ jukka.suomela@cs.helsinki.fi
}

\begin{abstract}
We study the following problem: given a geometric graph $\mathcal{G}$ and an integer $k$, determine if $\mathcal{G}$ has a planar spanning subgraph (with the original embedding and straight-line edges) such that all nodes have degree at least $k$. If $\mathcal{G}$ is a unit disk graph, the problem is trivial to solve for $k=1$. We show that even the slightest deviation from the trivial case (e.g., quasi unit disk graphs or $k=2$ ) leads to NP-hard problems.
\end{abstract}

\section{Introduction}

We study the problem of finding planar subgraphs that do not have low-degree nodes. More precisely, given a geometric graph $\mathcal{G}=(V, E)$ and an integer $k$, we want to determine if $\mathcal{G}$ has a planar spanning subgraph with the original embedding and straight-line edges such that all nodes have degree at least $k$.

This is a natural prerequisite in many problems related to communication networks. For example, if the answer is no, then certainly we cannot find a $k$-vertex connected or $k$-edge connected planar spanning subgraph, either. Moreover, if the answer is no, then we know that the domatic number (the maximum number of disjoint dominating sets) of any planar spanning subgraph is at most $k$. On the positive side, if the answer is yes for $k=1$, then we can find a planar spanning subgraph that has domatic number at least 2 . That is, we can partition the nodes in two disjoint dominating sets; for example, in a monitoring application, the two sets of nodes can take turns in order to conserve energy.

The problem is easy to solve if $\mathcal{G}$ is a complete graph and $k \leq 2$ : any triangulation of $V$ is a planar graph of degree at least 2, and hence the answer is yes iff we have at least $k+1$ nodes. The case of complete graphs and $k=3$ requires more thought, but it turns out that there is a planar spanning subgraph of minimum degree 3 iff we have at least 4 nodes and they are not in a convex position $[2,11]$. Hence the problem can be solved in polynomial time for complete graphs and $k \leq 3$. On the other hand, for $k \geq 6$ the answer is always no: any planar graph contains a node of degree at most 5 .

The problem is also easy to solve if $\mathcal{G}$ is a connected unit disk graph (see below for the definitions) and $k=1$ : the answer is yes if there are at least two nodes, since the Euclidean minimum spanning tree in $\mathcal{G}$ is planar. In this work we investigate what happens if we slightly deviate from the trivial case of a unit disk graph and $k=1$. In particular, can we solve the problem efficiently in quasi 
unit disk graphs [3], which are relaxations of unit disk graphs? Or can we solve the problem in other simple families of geometric graphs such as graphs with orthogonal edges? And what happens if $k=2$ or $k=3$ ? Surprisingly, it turns out that even the slightest deviations lead to NP-complete decision problems.

Related Work. To our knowledge, this problem has not been studied before, but there are related problems that have been considered in prior work.

In a non-geometric setting (i.e., the embedding of the nodes is not fixed), finding a planar spanning subgraph is trivial: any spanning tree is planar. However, finding large planar subgraphs is hard. For example, deciding if there is a planar subgraph with a certain number of edges is a classical NP-complete problem [5, problem GT27]. We refer to the survey by Liebers [8] for many variants of the theme.

Our focus is on a geometric setting: the input is a graph with a fixed embedding of the nodes, edges are straight lines, and a subgraph is planar if its edges do not cross each other. With these definitions, finding a planar spanning subgraph is no longer trivial: for example, an arbitrary spanning tree may have crossing edges. However, in unit disk graphs the problem is easy to solve: the Euclidean minimum spanning tree is planar. Indeed, a spanning tree is also connected, and many algorithms have been proposed to obtain a connected planar spanning subgraph of a unit disk graph - see, e.g., Gabriel and Sokal [4] and Toussaint [12]. However, none of these algorithms guarantee that the minimum degree is greater than one, and more general settings such as quasi unit disk graphs have not been extensively studied.

Another closely related problem is connectivity augmentation in which new edges are added to a graph to increase the connectivity. Abellanas et al. [1], Rutter and Wolff [9], and Tóth [10] consider geometric planar graphs and add edges to obtain 2-edge connected planar graphs, and Tóth and Valtr [11] and Al-Jubeh et al. [2] consider the problem of augmenting a geometric planar graph into a 3-edge connected planar graph. However, in these results the newly added edges are of unbounded length. Recently, Kranakis et al. [6] proved that a geometric planar graph can be augmented to a 2-edge connected planar graph with edges of length at most three times the largest edge in the graph.

Notation and Preliminaries. A geometric graph (straight line graph) $\mathcal{G}=$ $(V, E)$ is a graph where each $v \in V$ is a point in the Euclidean plane and each $e \in E$ is a straight line segment joining two distinct points. Throughout this paper we assume that the points are in general position (no three points being collinear). A geometric graph is planar if its edges do not cross each other except at their endpoints, and it is orthogonal if each edge is either horizontal or vertical. A graph is $k$-vertex connected if it remains connected after the removal of any $k-1$ vertices, and it is $k$-edge connected if it remains connected after the removal of any $k-1$ edges. The degree of node $v$ in graph $\mathcal{G}$ is denoted by $\operatorname{deg}(\mathcal{G}, v)$.

We define the family qUDG $(s)$ of quasi unit disk graphs with parameter $s \leq 1$ as follows. A geometric graph $\mathcal{G}=(V, E)$ is in $\operatorname{qUDG}(s)$ if the following holds for 
Table 1. Computational complexity of finding a planar spanning subgraph that has a given minimum degree in different families of geometric graphs. NP-C $=$ NP-complete.

\begin{tabular}{cllllc}
\hline Degree & Complete graphs & UDG & qUDG $(1-\epsilon)$ & Orthogonal graphs & Reference \\
\hline$\geq 1$ & in P & in P & NP-C if $\epsilon>0$ & NP-C & $\S 3$ \\
$\geq 2$ & in P & NP-C & NP-C if $\epsilon \geq 0$ & (open) & $\S 4$ \\
$\geq 3$ & in P $[11]$ & NP-C & NP-C if $\epsilon \geq 0$ & (open) & $\S 5$ \\
\hline
\end{tabular}

Table 2. Computational complexity of the augmentation problem.

\begin{tabular}{cllc}
\hline Degree & Graph family & Complexity & Reference \\
\hline$\geq 1$ & UDG & in P & \\
$\geq 2$ & UDG & NP-C if $\alpha<\sqrt{5} / 2$ & $\S 4$ \\
& UDG & in P if $\alpha \geq 3$ & {$[6]$} \\
& UDG, strip of height $h$ & in P if $\alpha \geq \sqrt{1+h^{2}}$ & $\S 6$ \\
$\geq 3$ & UDG & NP-C if $\alpha<\sqrt{5} / 2$ & $\S 5$ \\
\hline
\end{tabular}

all $u, v \in V$ : if the distance between $u$ and $v$ is at most $s$, then there is an edge $\{u, v\} \in E$, and if the distance between $u$ and $v$ is larger than 1 , then there is no edge between $u$ and $v$. The family UDG of unit disk graphs is equal to qUDG(1); in a unit disk graph, the locations of the nodes determine the set of edges.

If $\mathcal{G} \in$ UDG and $\alpha \geq 1$ is a real number, then we define the geometric $\alpha$ th power of a geometric graph $\mathcal{G}$, denoted by $\mathcal{G}^{(\alpha)}$, as the graph obtained from $\mathcal{G}$ by adding all edges between vertices of Euclidean distance at most $\alpha$.

Contributions. Our results are summarized in Tables 1 and 2 .

In Section 3 we study the case of minimum degree 1 . While the problem is trivial to solve in UDG, we show that it is NP-complete in qUDG $(1-\epsilon)$ for any positive constant $\epsilon$. We also show that the problem is NP-complete in orthogonal graphs. The case of $k=2$ is investigated in Section 4 , and the case of $k=3$ in Section 5. In both cases it turns out that the problem is NP-complete in UDG.

As the strict decision problem turns out to be NP-complete in UDG if $k \in$ $\{2,3\}$, it is natural to ask whether we can solve the augmentation version of the problem with parameter $\alpha \geq 1$ : either (i) prove that a given $\mathcal{G} \in$ UDG does not have a planar spanning subgraph of minimum degree $k$, or (ii) prove that $\mathcal{G}^{(\alpha)}$ has a planar subgraph of minimum degree $k$. The case of $\alpha=1$ is equivalent to the original decision problem. Our results in Sections 4 and 5 show that the case of $k \in\{2,3\}$ and $\alpha<\sqrt{5} / 2$ is NP-hard, while prior work [6] implies that the case of $k=2$ and $\alpha=3$ can be solved in polynomial time. In Section 6 we present an algorithm that solves the augmentation problem in narrow strips of height $h$ in polynomial time for $k=2$ and $\alpha=\sqrt{1+h^{2}}$.

The main new technique that we introduce in this work is the concept of planar circuit networks. In Section 2 we show that the problem of choosing an 
orientation of such a network is NP-complete, and Sections 3-5 demonstrate that the orientation problem on planar circuit networks serves as a useful starting point in NP-completeness proofs that are related to planar subgraphs.

\section{Orientation Problem on Planar Circuit Networks}

A planar circuit network is a planar geometric graph $\mathcal{C}=\left(V_{\mathcal{C}}, E_{\mathcal{C}}\right)$ with the following properties:

(i) The node set $V_{\mathcal{C}}=T_{\mathcal{C}} \cup S_{\mathcal{C}} \cup U_{\mathcal{C}}$ consists of three disjoint subsets: terminals $T_{\mathcal{C}}$, switches $S_{\mathcal{C}}$, and users $U_{\mathcal{C}}$.

(ii) The edge set $E_{\mathcal{C}}=P_{\mathcal{C}} \cup W_{\mathcal{C}}$ consists of two disjoint subsets: ports $P_{\mathcal{C}}$ and wires $W_{\mathcal{C}}$. Each port is labeled with either 1, 2, or 3 ; the set of ports with label $x$ is denoted by $P_{\mathcal{C}}(x)$.

(iii) Each wire joins a pair of terminals. Each port joins a terminal and a non-terminal.

(iv) Each terminal is incident to exactly 2 edges, and at least one of them is a wire.

(v) Each switch or user is incident to exactly 3 edges, and all of them are ports with different labels. That is, for each $v \in S_{\mathcal{C}} \cup U_{\mathcal{C}}$ and $x \in\{1,2,3\}$, there is exactly one edge in $P_{\mathcal{C}}(x)$ that is incident to $v$.

Refer to Fig. 1 for an illustration; we draw terminals as black dots, switches as trapezoids with the port 1 on the short side, and users as squares. In what follows, we use the notation $v(x)$ to refer to the unique port with label $x \in\{1,2,3\}$ that is incident to $v \in S_{\mathcal{C}} \cup U_{\mathcal{C}}$.

We can partition a circuit network into components that are connected to each other by terminals. More precisely, a component consists of (i) a wire and two terminals or (ii) a user or a switch, three ports, and three terminals. Note that each terminal is contained in exactly two components. However, if $a$ is a wire, switch, or user, then there is a unique component $\mathcal{C}[a]$ that contains $a$; in that case, we use the notation $a \bullet t$ to denote that $t$ is a terminal in the component $\mathcal{C}[a]$. We write $a \stackrel{x}{\bullet} t$ if the port that leads from switch or user $a$ to terminal $t$ has label $x$.

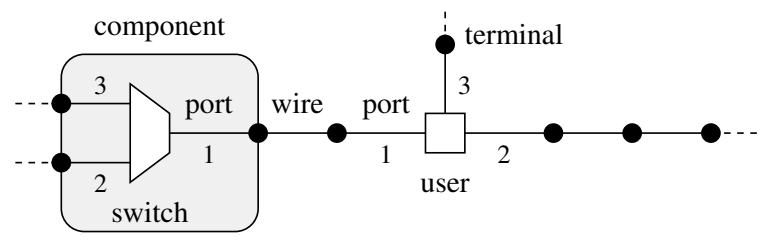

Fig. 1. A circuit network. 
(a)

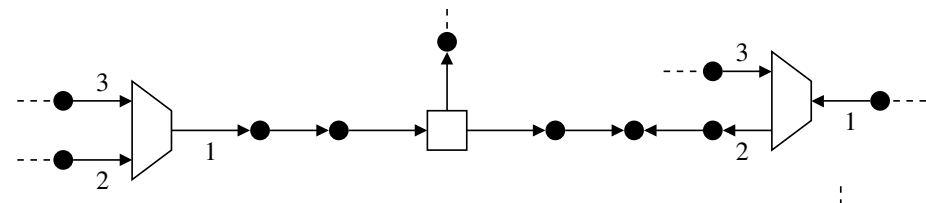

(b)
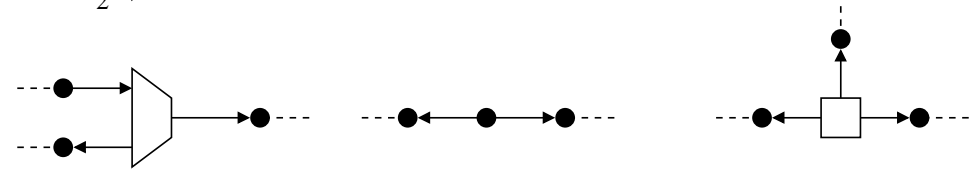

Fig. 2. (a) Valid and (b) invalid orientations of a circuit network.

Orientation Problem. An orientation of a planar circuit network $\mathcal{C}$ assigns a direction to each edge $e \in E_{\mathcal{C}}$. If $a \rightarrow t$, and $t$ has indegree 1 in the component $\mathcal{C}[a]$, then we write $a \rightarrow t$; otherwise $a \triangleleft \bullet t$. If $a \stackrel{x}{\bullet} t$, we use notation $a \stackrel{x}{\bullet} t$ or $a \triangleleft x$

A valid orientation of a component $\mathcal{C}[a]$ satisfies the following requirements (the first one is trivially satisfied but is listed here for reference):

(i) If $a$ is a wire, then there is at least one terminal $t$ with $a \triangleleft \bullet t$.

(ii) If $a$ is a user, then there is at least one terminal $t$ with $a \triangleleft \bullet t$.

(iii) If $a$ is a switch with $s \stackrel{2}{\longrightarrow} t_{2}$ or $s \stackrel{3}{\longrightarrow} t_{3}$, then $s \stackrel{1}{\bullet} t_{1}$.

A valid orientation of a circuit network $\mathcal{C}$ satisfies the following additional requirement:

(iv) Each terminal has indegree at least one.

See Fig. 2 for an illustration. Not all circuit networks have valid orientations; in the orientation problem the task is to decide if a given planar circuit network $\mathcal{C}$ has a valid orientation.

The following lemma shows that we can replace a path of wires by a single wire and vice versa, without affecting the essential properties of the problem.

Lemma 1. Let $\mathcal{C}$ be a planar circuit network, and let $e=\{u, v\} \in W_{\mathcal{C}}$ be a wire in $\mathcal{C}$. Construct another planar circuit network $\mathcal{C}^{\prime}$ by repeatedly subdividing $e$; that is, we replace $e$ by a path $P$ that consists of wires and terminals. Then if we are given a valid orientation of $\mathcal{C}$, we can find in polynomial time a valid orientation of $\mathcal{C}^{\prime}$, and vice versa.

Proof. Clearly if we are given a valid orientation of $\mathcal{C}$, we can construct a valid orientation of $\mathcal{C}^{\prime}$ as well. Now assume that we are given a valid orientation of $\mathcal{C}^{\prime}$. Since all internal nodes of $P$ have indegree at least 1 , there must be at least one endpoint of $P$ that has indegree 0 . W.l.o.g., we can assume that $u$ is an endpoint with indegree 0 . Then we can orient the wire $e$ in $\mathcal{C}$ from $u$ to $v$. The orientations of all other edges are inherited from $\mathcal{C}^{\prime}$.

We will use the following theorem in reductions throughout this work. 
Theorem 1. The orientation problem on planar circuit networks is NP-complete.

Proof. By a reduction from planar 3SAT; see the extended version of this work [7].

\section{Degree One}

In this section we study the problem of deciding if a given geometric graph has a planar spanning subgraph. The problem is trivial in UDG - a minimum spanning tree is a planar spanning subgraph. We will show that the problem is NP-complete in quasi unit disk graphs and in orthogonal graphs.

Theorem 2. The following problem is NP-complete for any $\epsilon>0$ : given a graph $\mathcal{G} \in \mathrm{qUDG}(1-\epsilon)$, decide if $\mathcal{G}$ has a planar spanning subgraph.

To prove the theorem, assume that we are given an $\epsilon>0$ and a planar circuit network $\mathcal{C}$. We will show how to construct a graph $\mathcal{G} \in \operatorname{qUDG}(1-\epsilon)$ with the following property: $\mathcal{G}$ has a planar spanning subgraph if and only if $\mathcal{C}$ has a valid orientation. The claim then follows by Theorem 1 .

In our construction of $\mathcal{G}$, we replace each component $\mathcal{C}[a]$ of $\mathcal{C}$ by a gadget $\mathcal{G}[a]$ that implements the component. In the construction, we will have two kinds of nodes in $\mathcal{G}$ : black nodes are identified with the terminals of $\mathcal{C}$, while grey nodes are internal to a gadget. A component with a wire is replaced with the gadget of Fig. 3a, a component with a switch is replaced with the gadget of Fig. 3b, and a component with a switch is replaced with the gadget of Fig. 3c. Note that each black node is shared by exactly two gadgets.

To guarantee that the gadgets are in qUDG $(1-\epsilon)$, we must choose appropriate dimensions. Moreover, two gadgets must not be placed too close to each other; for example, an internal node of one gadget cannot be within distance $1-\epsilon$

(a)
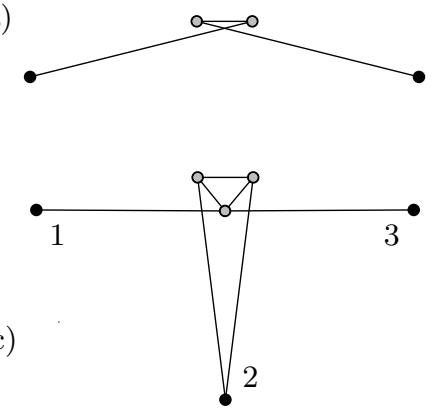

(b)

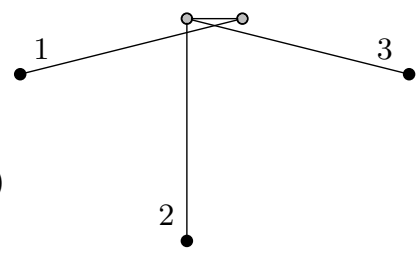

(d)

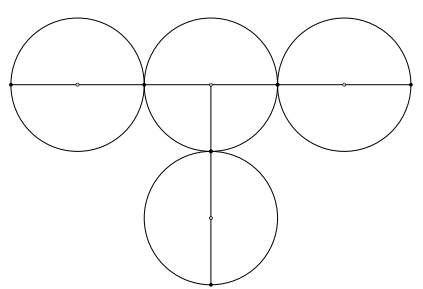

Fig. 3. Gadgets for qUDG $(1-\epsilon)$ and minimum degree 1: (a) wire, (b) switch, (c) user. (d) Embedding of a switch, three ports, and three wires. 
from the internal node of another gadget. Hence we cannot directly replace the components by gadgets in an arbitrary embedding of $\mathcal{C}$. However, we can always find an appropriate embedding by moving the components around and by exploiting the flexibility provided by Lemma 1; see Fig. 3d for an illustration of the embedding in the neighborhood of a switch. Note that we can use a polynomially-sized grid to embed the nodes, as we have some flexibility in the choice of the exact coordinates: for example, in the wire gadget, the distance between the two terminals can be chosen from the range $(2-\epsilon, 2)$.

Now we proceed to relate the orientations of $\mathcal{C}$ and the planar spanning subgraphs of $\mathcal{G}$. Let us first focus on a component $\mathcal{C}[a]$ and the gadget $\mathcal{G}[a]$ that implements it. We say that a subgraph $\mathcal{G}^{\prime}[a]$ of $\mathcal{G}[a]$ is internally good if $\mathcal{G}^{\prime}[a]$ is planar and each grey node of $\mathcal{G}[a]$ has degree at least one in $\mathcal{G}^{\prime}[a]$. Note that if we have a planar spanning subgraph $\mathcal{G}^{\prime}$ of $\mathcal{G}$, then $\mathcal{G}^{\prime}$ restricted to $\mathcal{G}[a]$ is internally good. The key observation is summarized in the following lemma.

Lemma 2. Given a valid orientation of $\mathcal{C}[a]$, we can find an internally good subgraph $\mathcal{G}^{\prime}[a]$ such that $a \rightarrow t$ implies $\operatorname{deg}\left(\mathcal{G}^{\prime}[a], t\right) \geq 1$. Conversely, given an internally good subgraph $\mathcal{G}^{\prime}[a]$, we can find a valid orientation of $\mathcal{C}[$ a] such that $a \bullet t$ and $\operatorname{deg}\left(\mathcal{G}^{\prime}[a], t\right) \geq 1$ implies $a \rightarrow b$.

Proof. A straightforward case analysis.

Now if we are given a valid orientation of $\mathcal{C}$, we can apply Lemma 2 to each component $\mathcal{C}[a]$ to find an internally good subgraph $\mathcal{G}^{\prime}[a]$ for each $\mathcal{G}[a]$; the union of $\mathcal{G}^{\prime}[a]$ forms a subgraph $\mathcal{G}^{\prime}$ of $\mathcal{G}$. By construction, $\mathcal{G}^{\prime}$ is planar and each grey node has degree at least one. It remains to be shown that each black node has degree at least one. To verify this, consider a terminal $t$. In a valid orientation, there is a component $\mathcal{C}[a]$ such that $a \rightarrow t$. Hence in $\mathcal{G}^{\prime}[a]$ we have $\operatorname{deg}\left(\mathcal{G}^{\prime}[a], t\right) \geq 1$, and therefore also $\operatorname{deg}\left(\mathcal{G}^{\prime}, t\right) \geq 1$. Hence $\mathcal{G}^{\prime}$ is a planar spanning subgraph of $\mathcal{G}$.

Conversely, if we are given a planar spanning subgraph $\mathcal{G}^{\prime}$ of $\mathcal{G}$, we can apply Lemma 2 to each component $\mathcal{G}[a]$ to orient $\mathcal{C}$. We will have a valid orientation for each component; it remains to be shown that each terminal has indegree at least one. To verify this, consider a terminal $t$. Since $\operatorname{deg}\left(\mathcal{G}^{\prime}, t\right) \geq 1$ we have a component $\mathcal{C}[a]$ such that $\operatorname{deg}\left(\mathcal{G}^{\prime}[a], t\right) \geq 1$, and hence a valid orientation with $a \rightarrow t$. Hence we have a valid orientation of $\mathcal{C}$. This concludes the proof of Theorem 2 .

Theorem 3. The following problem is NP-complete: given an orthogonal graph $\mathcal{G}$, decide if it has a planar spanning subgraph.

Proof. The structure of the proof is identical to the proof of Theorem 2. We are only using a different set of gadgets: see Fig. 4.

\section{Degree Two}

In this section we study the case of planar spanning subgraphs that have degree at least 2. It turns out that finding such subgraphs is NP-hard even in the case of UDG. 

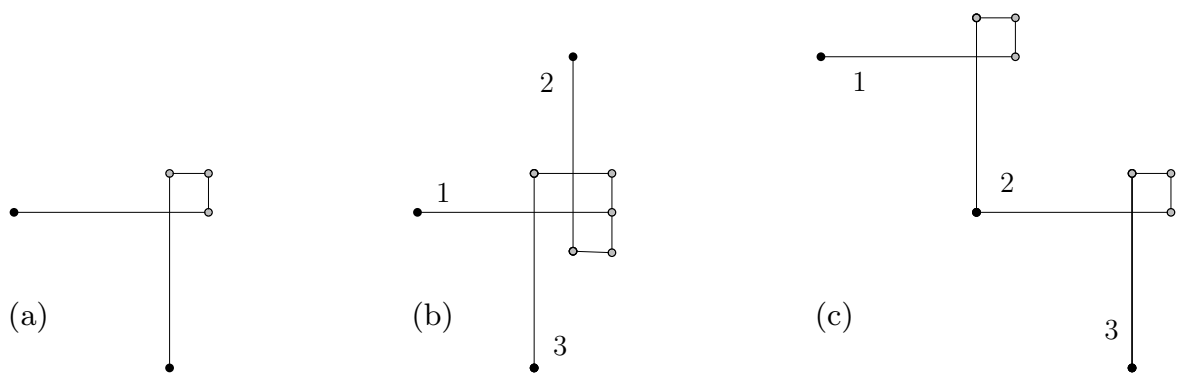

Fig. 4. Gadgets for orthogonal graphs: (a) wire, (b) switch, (c) user.

In what follows, we prove a stronger result by considering a variant in which we are allowed to augment the graph by adding edges of length $\alpha \geq 1$. In the augmentation problem we are allowed to return the answer that "the original graph does not have a planar spanning subgraph with minimum degree at least 2 " or "I do not know about the original graph, but if I first add some edges of length $\alpha, I$ can construct a planar spanning subgraph with minimum degree at least 2 ". Obviously, the case of $\alpha=1$ is equivalent to the original decision problem.

If $\alpha=3$, the problem can be solved in polynomial time by using techniques from prior work [6]: If we are given a graph $\mathcal{G} \in$ UDG, we can consider each connected component of $\mathcal{G}$ separately. If a connected component contains fewer than 3 nodes, then we know that $\mathcal{G}$ does not have a planar subgraph with minimum degree 2. Otherwise we can first find a spanning tree in each component, and then augment the trees by adding non-crossing edges of length at most 3 so that each connected component becomes 2-edge connected. In particular, after augmentation, the graph is planar and each node has minimum degree at least 2 .

In what follows, we prove that the problem is NP-complete if $\alpha<\sqrt{5} / 2 \approx$ 1.118. We do this by formulating the problem as a promise problem.

Theorem 4. The following promise problem is NP-complete for any $1 \leq \alpha<$ $\sqrt{5} / 2$ : given a graph $\mathcal{G} \in \mathrm{UDG}$, decide whether (i) there is no planar spanning subgraph of $\mathcal{G}$ such that each node has degree at least 2 , or (ii) there is a planar spanning subgraph of $\mathcal{G}^{(\alpha)}$ such that each node has degree at least 2.

Proof. The structure of the proof is similar to the proof of Theorem 2. For the sake of brevity, we only list the differences.

In Theorem 2 we constructed a graph $\mathcal{G} \in$ qUDG $(1-\epsilon)$; this time we construct a graph $\mathcal{G} \in$ UDG. Moreover, our construction satisfies $\mathcal{G}=\mathcal{G}^{(\alpha)}$, that is, the augmentation does not change the graph at all, and hence the augmentation problem is exactly as difficult as deciding if $\mathcal{G}$ has a planar spanning subgraph with minimum degree at least two.

We use a new set of gadgets; see Fig. 5. In addition to the gadgets that correspond to the components of the circuit network, we also have a gadget for each terminal. It can be verified that the distance between any pair of non- 

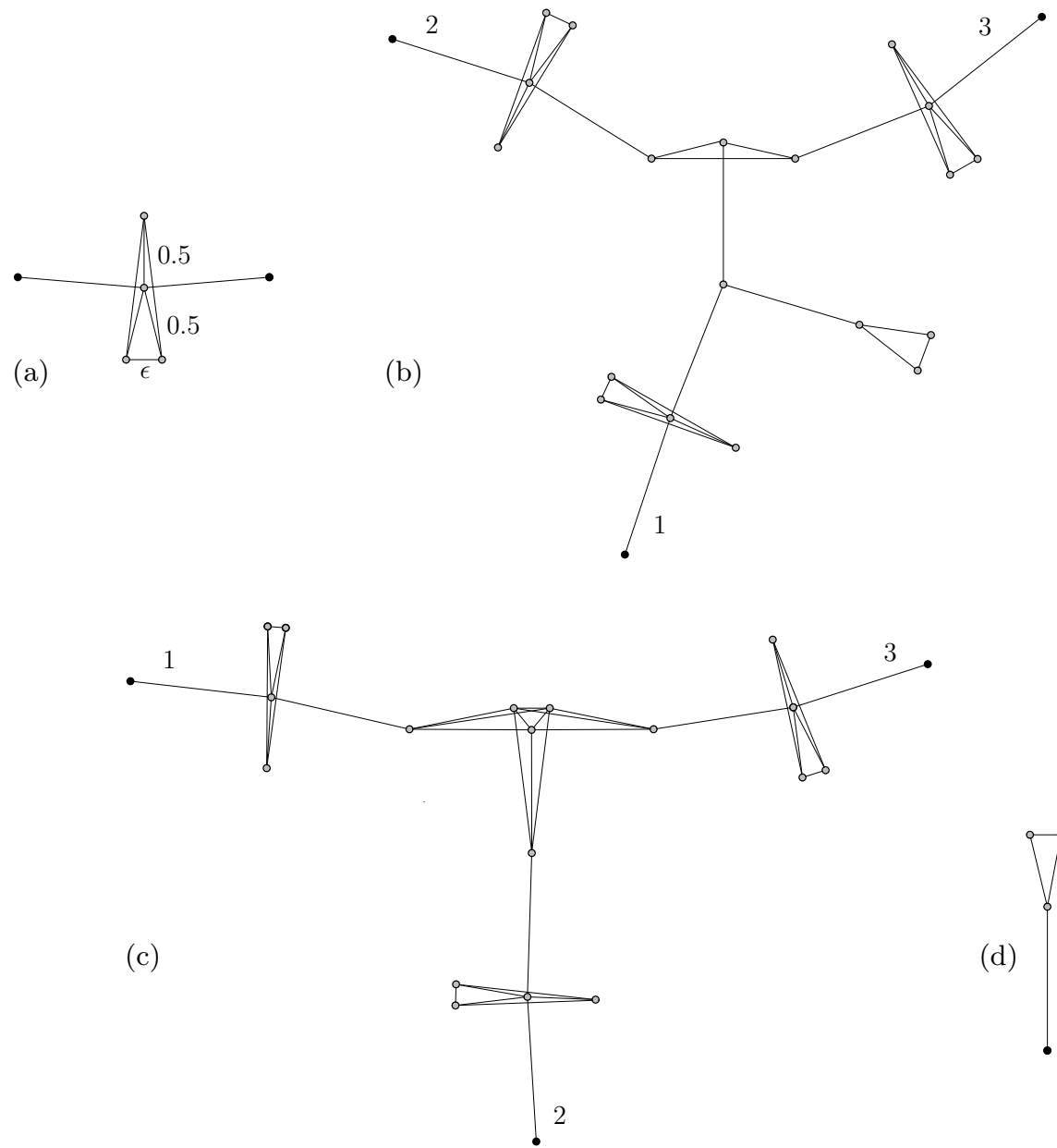

(d)

Fig. 5. Gadgets for UDG, minimum degree 2: (a) wire, (b) switch, (c) user, (d) terminal.

adjacent nodes within a gadget is larger than $\alpha$, and that there is an embedding that preserves this property.

As we are finding a subgraph of minimum degree 2, we change the definition of an internally good subgraph accordingly: each grey node must have degree at least 2 . With this change, Lemma 2 holds verbatim.

The key difference with the proof of Theorem 2 is that we have to deal with the terminal gadgets. If we are given a valid orientation of $\mathcal{C}$, we can apply Lemma 2 to each component $\mathcal{C}[a]$ to find an internally good subgraph $\mathcal{G}^{\prime}[a]$ for each $\mathcal{G}[a]$. The union of the subgraphs $\mathcal{G}^{\prime}[a]$ and all terminal gadgets forms a subgraph $\mathcal{G}^{\prime}$ of $\mathcal{G}$. By construction, $\mathcal{G}^{\prime}$ is planar and each grey node has degree at least 2 . Now consider a terminal $t$. In a valid orientation, there is a component $\mathcal{C}[a]$ such that $a \rightarrow t$, and we have $\operatorname{deg}\left(\mathcal{G}^{\prime}[a], t\right) \geq 1$. Furthermore, $t$ is incident to 
exactly one edge from the terminal gadget. In summary, $\operatorname{deg}\left(\mathcal{G}^{\prime}, t\right) \geq 2$. Hence $\mathcal{G}^{\prime}$ is a planar spanning subgraph of $\mathcal{G}$ and all nodes have degree at least 2

Conversely, given a planar spanning subgraph $\mathcal{G}^{\prime}$ with minimum degree 2 , we can verify that we can construct a valid orientation of $\mathcal{C}$ : each terminal gadget contributes only one edge, and hence for each terminal $t$ we must have $a \rightarrow b t$ such that $\operatorname{deg}\left(\mathcal{G}^{\prime}[a], t\right) \geq 1$. This concludes the proof.

\section{Degree Three}

In this section, we consider the case of planar spanning subgraphs with minimum degree at least $k=3$. This case turns out to be similar to that of $k=2$ in Section 4. Again, we can prove that the problem is NP-complete, and even the augmentation version of the problem is NP-complete.

Theorem 5. The following promise problem is NP-complete for any $1 \leq \alpha<$ $\sqrt{5} / 2$ : given a graph $\mathcal{G} \in \mathrm{UDG}$, decide whether (i) there is no planar spanning subgraph of $\mathcal{G}$ such that each node has degree at least 2 , or (ii) there is a planar spanning subgraph of $\mathcal{G}^{(\alpha)}$ such that each node has degree at least 2.

Proof. The proof is similar to Theorem 2. The key differences are as follows: We have a new set of gadgets, see Fig. 6 . We do not have any terminal gadgets. The definition of an internally good subgraph is changed accordingly: each grey node has degree at least 3. Finally, Lemma 2 is modified as follows.
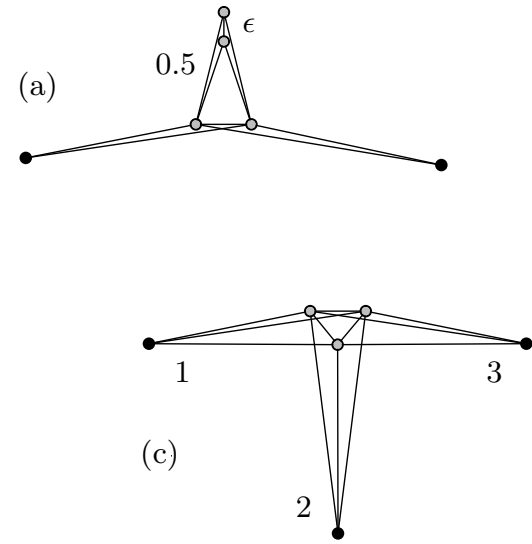

(b)

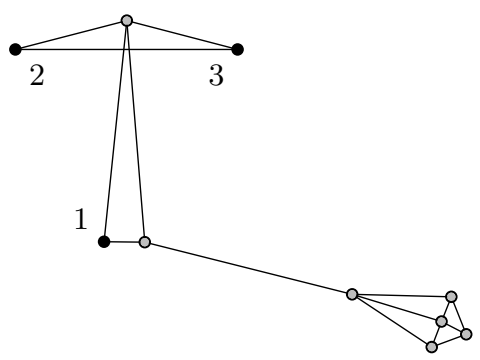

(d)

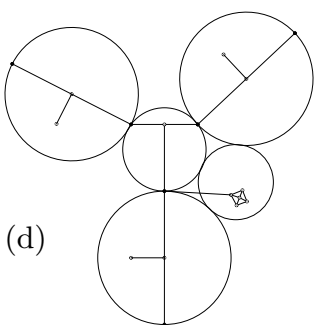

Fig. 6. Gadgets for UDG and minimum degree 3: (a) wire, (b) switch, (c) user. (d) Embedding of a switch and three wires. 
Lemma 3. Given a valid orientation of $\mathcal{C}[a]$, we can find an internally good subgraph $\mathcal{G}^{\prime}[a]$ such that $a \rightarrow t$ implies $\operatorname{deg}\left(\mathcal{G}^{\prime}[a], t\right) \geq 1$ and $a \rightarrow \bullet$ implies $\operatorname{deg}\left(\mathcal{G}^{\prime}[a], t\right)$ $\geq 2$. Conversely, given an internally good subgraph $\mathcal{G}^{\prime}[a]$, we can find a valid orientation of $\mathcal{C}[a]$ such that $a \rightarrow t$ and $\operatorname{deg}\left(\mathcal{G}^{\prime}[a], t\right) \geq 2$ implies $a \rightarrow \bullet t$.

Now if we are given a valid orientation of $\mathcal{C}$, we can apply Lemma 3 to each component in order to construct a planar subgraph $\mathcal{G}^{\prime}$ of $\mathcal{G}$. For each terminal $t$ there are $a \rightarrow t$ and $b \bullet t$ with $a \neq b$. Now $\operatorname{deg}\left(\mathcal{G}^{\prime}, t\right)=\operatorname{deg}\left(\mathcal{G}^{\prime}[a], t\right)+$ $\operatorname{deg}\left(\mathcal{G}^{\prime}[b], t\right) \geq 2+1=3$. The converse case is similar.

\section{Augmenting with Bounded Length Edges}

As we saw in Section 4, the augmentation problem for the case of minimum degree $k=2$ is NP-complete in UDG if we are allowed to augment by adding edges of length $\alpha<\sqrt{5} / 2$, and it can be solved in polynomial time if $\alpha=3$. In this section we present an algorithm that solves the augmentation problem for $\alpha=\sqrt{1+h^{2}}$ in narrow strips of height $h$.

Theorem 6. The following problem can be solved in polynomial time: given a graph $\mathcal{G} \in$ UDG in which all nodes are inside a strip of height $h$, either (i) show that there is no planar spanning subgraph of $\mathcal{G}$ such that each node has degree at least 2 , or (ii) find a planar spanning subgraph of $\mathcal{G}^{(\alpha)}$ for $\alpha=\sqrt{1+h^{2}}$ such that each node has degree at least 2.

Proof. Let $V=\left\{v_{1}, v_{2}, \ldots, v_{n}\right\}$ be the nodes of $\mathcal{G}$ ordered by their $x$-coordinates, and let $x_{i}$ be the $x$-coordinate of node $v_{i}$; that is, we have $x_{i} \leq x_{i+1}$ for all $i<n$. We say that $V_{s, t}=\left\{v_{s}, v_{s+1}, \ldots, v_{t}\right\}$ is a section if

$$
\begin{aligned}
& -x_{i}+1 \geq x_{i+1} \text { for all } s \leq i<t, \\
& -s=1 \text { or } x_{s-1}+1<x_{s}, \text { and } \\
& -t=n \text { or } x_{t}+1<x_{t+1} .
\end{aligned}
$$

Put otherwise, a section is a maximal set of nodes such that the $x$ coordinates are separated by at most 1 unit. Note that the subgraph $\mathcal{G}_{s, t}$ induced by section $V_{s, t}$ consists of one or more connected components of $\mathcal{G}$.

Now consider each subgraph $\mathcal{G}_{s, t}$ one by one; we will either conclude that we have case (i), or we will show how find a planar subgraph $\mathcal{G}_{s, t}^{\prime}$ of $\mathcal{G}_{s, t}^{(\alpha)}$ :

- If $t-s \leq 5$, we have a constant-size subproblem that we can solve by brute force: either decide that we have case (i), or construct a planar subgraph $\mathcal{G}_{s, t}^{\prime}$.

- If $x_{s}+1<x_{s+2}$, then the degree of $v_{s}$ in $\mathcal{G}$ is at most one, and we have case (i). Similarly, if $x_{t-2}+1<x_{t}$, then the degree of $v_{t}$ in $\mathcal{G}$ is at most one, and we have case (i).

- Otherwise we can construct $\mathcal{G}_{s, t}^{\prime}$ as follows: the edge set of $\mathcal{G}_{s, t}^{\prime}$ consists of $\left\{v_{s}, v_{s+2}\right\},\left\{v_{t-2}, v_{t}\right\}$, and $\left\{v_{i}, v_{i+1}\right\}$ for all $s \leq i<t$; each of these has length at most $\alpha$, and they are nonintersecting.

If we do not have case (i), we can find a planar subgraph of $\mathcal{G}^{(\alpha)}$ as a union of the subgraphs $\mathcal{G}_{s, t}^{\prime}$. 


\section{Conclusions}

In this work we have studied the problem of finding a planar spanning subgraph with minimum degree $k$ in different families of geometric graphs. One of the main discoveries is the existence of a very sharp threshold in the computational complexity of such problems: the case of $k=1$ and unit disk graphs is trivial, while a minor deviation from $k=1$ to $k=2$, or from unit disk graphs to quasi unit disk graphs makes the problem NP-complete. A major open problem is a full characterization of the complexity of the augmentation problem: for which values of the parameter $\alpha$ can the problem be solved in polynomial time?

Acknowledgements. We thank the anonymous reviewers for their helpful comments and suggestions. This work was supported in part by NSERC, MITACS, CONACYT, the Academy of Finland (Grant 132380), the Finnish Cultural Foundation, and the Research Funds of the University of Helsinki.

\section{References}

1. Abellanas, M., García, A., Hurtado, F., Tejel, J., Urrutia, J.: Augmenting the connectivity of geometric graphs. Computational Geometry: Theory and Applications 40(3), 220-230 (2008)

2. Al-Jubeh, M., Ishaque, M., Rédei, K., Souvaine, D.L., Tóth, C.D.: Tri-edgeconnectivity augmentation for planar straight line graphs. In: Proc. 20th International Symposium on Algorithms and Computation (ISAAC 2009). LNCS, vol. 5878, pp. 902-912. Springer (2009)

3. Barrière, L., Fraigniaud, P., Narayanan, L., Opatrny, J.: Robust position-based routing in wireless ad hoc networks with irregular transmission ranges. Wireless Communications and Mobile Computing 3(2), 141-153 (2003)

4. Gabriel, K.R., Sokal, R.R.: A new statistical approach to geographic variation analysis. Systematic Zoology 18(3), 259-278 (1969)

5. Garey, M.R., Johnson, D.S.: Computers and Intractability: A Guide to the Theory of NP-Completeness. WH Freeman and Company (1979)

6. Kranakis, E., Krizanc, D., Morales Ponce, O., Stacho, L.: Bounded length, 2edge augmentation of geometric planar graphs. In: Proc. 4th Annual International Conference on Combinatorial Optimization and Applications (COCOA 2010). LNCS, vol. 6509, pp. 385-397. Springer (2010)

7. Kranakis, E., Morales Ponce, O., Suomela, J.: Planar subgraphs without low-degree notes. http://www.iki.fi/jukka.suomela/low-degree (2011)

8. Liebers, A.: Planarizing graphs - a survey and annotated bibliography. Journal of Graph Algorithms and Applications 5(1), 1-74 (2001)

9. Rutter, I., Wolff, A.: Augmenting the connectivity of planar and geometric graphs. Electronic Notes in Discrete Mathematics 31, 53-56 (2008)

10. Tóth, C.D.: Connectivity augmentation in plane straight line graphs. Electronic Notes in Discrete Mathematics 31, 49-52 (2008)

11. Tóth, C.D., Valtr, P.: Augmenting the edge connectivity of planar straight line graphs to three. In: Proc. 13th Spanish Meeting on Computational Geometry (EGC 2009) (2009)

12. Toussaint, G.T.: The relative neighbourhood graph of a finite planar set. Pattern Recognition 12(4), 261-268 (1980) 